\title{
HVMANITAS
}

\section{Talete sophos (ad Atene, sotto l'arcontato di Damasia)}

Autor(es): Rossetti, Livio

Publicado por: Faculdade de Letras da Universidade de Coimbra, Instituto de Estudos

URL

persistente: URI:http://hdl.handle.net/10316.2/8525

DOI: $\quad$ DOI:http://dx.doi.org/10.14195/2183-1718_62_3

Accessed : $\quad$ 26-Apr-2023 04:27:32

A navegação consulta e descarregamento dos títulos inseridos nas Bibliotecas Digitais UC Digitalis, UC Pombalina e UC Impactum, pressupõem a aceitação plena e sem reservas dos Termos e Condições de Uso destas Bibliotecas Digitais, disponíveis em https://digitalis.uc.pt/pt-pt/termos.

Conforme exposto nos referidos Termos e Condições de Uso, o descarregamento de títulos de acesso restrito requer uma licença válida de autorização devendo o utilizador aceder ao(s) documento(s) a partir de um endereço de IP da instituição detentora da supramencionada licença.

Ao utilizador é apenas permitido o descarregamento para uso pessoal, pelo que o emprego do(s) título(s) descarregado(s) para outro fim, designadamente comercial, carece de autorização do respetivo autor ou editor da obra.

$\mathrm{Na}$ medida em que todas as obras da UC Digitalis se encontram protegidas pelo Código do Direito de Autor e Direitos Conexos e demais legislação aplicável, toda a cópia, parcial ou total, deste documento, nos casos em que é legalmente admitida, deverá conter ou fazer-se acompanhar por este aviso. 
humanitas

Vol. LXII

2010 


\title{
TALETE SOPHOS (AD ATENE, SOTTO L'ARCONTATO DI DAMASIA)
}

\author{
LiVIO RosSETTI \\ Università di Perugia
}

\begin{abstract}
È normale trattare come irrilevante la notizia secondo cui gli ateniesi conferirono a Talete il titolo di sophos, e in connessione con questa circostanza designarono i Sette Sapienti, ma l'argomento merita di essere riesaminato, poiché ci sono non pochi indizi per pensare che qualcosa del genere possa essere effettivamente accaduto. Questo articolo è dedicato all'esplorazione delle evidenze pertinenti.

Parole-chiave: Talete, Solone, Damasia, Sette Sapienti, Diogene Laerzio, sophos.
\end{abstract}

\section{Abstract}

It is customary to dismiss as irrelevant the news that Athenians awarded Thales with the title of sophos, and in connection with this event appointed the Seven Wise; however there is ample room for reconsidering the matter, since several clues are there to suggest that something of the kind is likely to have occurred. This paper is devoted to explore the relevant evidence.

Key-words: Thales, Solon, Damasias, Seven Wise, Diogenes Laertius, sophos.

In I 22 Diogene Laerzio cita la Archonton anagraphe di Demetrio

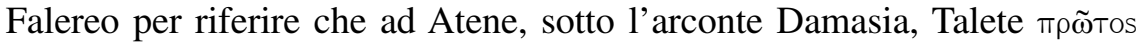

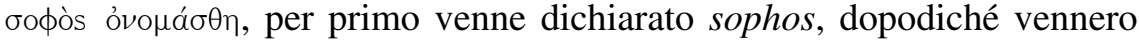
scelti anche gli altri intellettuali che formarono il gruppo noto come oi

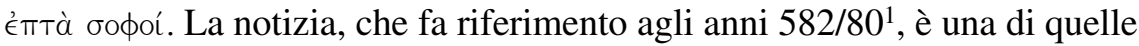

1 Questo perché il nome di Damasia è associato a una anomalia, consistente nel permanere nella carica di arconte per un secondo anno e per i primi due mesi di un terzo 
che vengono tradizionalmente ignorate, come se fosse priva di qualsiasi rilevanza, e sarà interessante vedere se qualcosa accadrà in questa fase in cui la figura di Talete ha attirato una rinnovata attenzione ${ }^{2}$.

Un buon motivo per interrogarsi sulla sua supposta irrilevanza prende forma se consideriamo che non sembra trattarsi di una notizia idonea ad essere inventata partendo da zero. Infatti nel periodo indicato, Atene era ancora lontana dall'essere diventata un centro culturale primario dell'ecumene ellenica e dal costituire addirittura «un ammaestramento per tutta la Grecia» (Thuc. 41.1). Già questo dato dissuade dal supporre che la storia possa essere stata inventata ex novo per poi essere trattata come un fatto realmente accaduto ${ }^{3}$. Ma non meno irrituali appaiono sia l'eventualità che Talete si sia recato ad Atene per essere proclamato sophos e ricevere una sorta di onorificenza, sia la notizia secondo cui quella di sophos venne trattata, per una volta, come una qualifica ambita, che si poteva attribuire - o negare - a un certo intellettuale. Di conseguenza ha senso disporsi a pensare che la notizia non sia inventata ma fondata su dei fatti su cui accendere, se possibile, i riflettori.

Dobbiamo subito chiederci se la cronologia ci assiste o, al contrario, fa problema. L'argomento è affrontato su questa stessa rivista nell'articolo del Prof. Leão. A me sembra di poter dire che i riscontri cronologici sono incoraggianti perché ci fu un Damasia arconte eponimo all'epoca che qui

anno, quando venne deposto a seguito di una rivolta, con la quale gli ateniesi seppero evitare la trasformazione di Damasia in tyrannos. In proposito si veda l'articolo di Delfim Leão in questo stesso fascicolo di Humanitas.

2 Ricordo O'Grady 2002, Moscarelli 2005, Wöhrle 2009; sono annunciati inoltre una specifica monografia di Andreas Schwab e una di Flavia Marcacci, ma per tutto il resto del secolo XX non si ricorda nulla di comparabile a un così alto livello di attenzione. Con l'occasione ricordo inoltre che si deve a M. M. Sassi (Sassi 2009, 60-63) un primo, meditato contributo volto a far luce sulla dimenticata parentesi ateniese di Talete.

3 Ragioni analoghe vengono addotte a buon diritto, io credo, a proposito dei dubbi sulla possibilità che abbia realmente avuto luogo la previsione dell'eclisse del 585 . In contrario è stata addotta la durevole propensione dell'opinione pubblica greca a vivere l'eclisse come un fenomeno straordinario e, per definizione, destabilizzante, quindi come un messaggio divino. Così la interpretarono, come è noto, anche alcuni strateghi ateniesi durante la guerra del Peloponneso (Plut. Nic. 23). Ciò costituisce un buon indizio per pensare che a un greco non sarebbe potuto venire in mente né di spiegarsi l'eclisse come effetto della mera interposizione di un corpo opaco (la luna) davanti al disco solare, né di inventare la storia del "dottorato honoris causa" che Atene avrebbe conferito a Talete e ad altri 'intellettuali'. 
viene richiamata, mentre il solo altro Damasia che risulta essere stato arconte eponimo lo fu nell'anno 639/38 a.C., dunque in tutt'altra epoca. Che cosa ci dice, invece, il 582/81? Anzitutto che la strepitosa previsione di una eclisse di sole era avvenuta da appena tre anni ed aveva dunque avuto solo il tempo necessario per diventare una notizia condivisa, risaputa, memorabile (per cui non è stravagante immaginare che la notizia abbia avuto un peso decisivo nell'indurre Atene ad onorare il genio di Talete). La data ci dice, in secondo luogo, che Solone era da poco rientrato dal suo volontario esilio, ossia dal decennio di viaggi a sud e soprattutto a est dell'Ellade propriamente detta. Osservo inoltre che la lista dei residui cinque sapienti che varie fonti tramandano ${ }^{4}$ è tale da far pensare che, oltre a Talete e a Solone, siano stati onorati dei personaggi di ben più modesta caratura, come se il processo di selezione dei candidati al titolo, pur sempre ambito, di sophos avesse subìto un rapido degrado.

Si delinea, con ciò, un insieme piuttosto congruente di informazioni, per cui sul fatto diventa difficile dubitare, e sul senso del fatto è a dir poco attraente spingersi a pensare che, di ritorno dai suoi viaggi nell'area anatolica (e quindi anche nella Ionia), Solone possa aver preso l'iniziativa di proporre che si onorasse il grande Talete e che Atene gli tributasse un riconoscimento pubblico e significativo, e anche di evitare che questo pubblico riconoscimento rimanesse un evento isolato, ma si ripetesse a favore di altri, dando vita ad una sorta di collegio dei sette sapenti, tra i quali includere se stesso. Naturalmente è ben difficile stabilire se l'idea è stata lanciata da Solone o si è trattato di una autonoma iniziativa di Damasia, e a maggior ragione se i sette sapienti vennero designati tutti in una volta o con cadenza annuale, ma questi sono dettagli molto secondari, perché la cosa importante è capire se il fatto avvenne, se cioè una iniziativa della polis ateniese, volta a onorare anzitutto Talete, sia stata effettivamente presa mentre Damasia era arconte eponimo.

Un ulteriore indizio a favore dell'attendibilità di tale notizia sembra provenire dall'eventualità che la notorietà di Talete sia stata relativamente grande, e grande anzitutto per l'assenza di altri intellettuali che fossero altrettanto celebrati. In effetti, l'eccellenza raggiunta da Talete nel sapere

4 Ricordo che in Prot. 343a Platone menziona Pittaco, Biante, Cleobulo, Chilone e Misone; che Plutarco (Symp. VIII Sap.) menziona invece Biante, Anacarsi, Cleobulo, Pittaco e Chilone, mentre Diogene Laerzio (I 13) menziona Periandro, Cleobulo, Chilone, Biante e Pittaco. 
- più precisamente nell' accesso a delle conoscenze formalmente inutili, ma comprensibili e, quel che più conta, in grado di entusiasmare - ha costituito un unicum per l'epoca. Infatti, nei cento anni che seguirono, si affermarono intellettuali come Anassimandro, Anassimene, Ecateo, Pitagora, Alcmeone di Crotone, Brotino o Brontino di Metaponto, Senofane, Eraclito ma, con la sola eccezione del suo allievo diretto Anassimandro, tutti in epoca successiva, per cui di nessuno di costoro si può immaginare che fosse già celebre ai tempi dell' arconte Damasia, così celebre di poter essere collocato sullo stesso piano di Talete. D'altra parte non è un caso che nessuno degli altri sei sapienti onorati ad Atene sia noto per essere stato portatore di un sapere peri physeos in qualche misura comparabile. Viceversa Talete è detto aver entusiasmato un certo Mandrolito di Priene per l'impensata possibilità di accedere a una modalità di investigazione in grado di condurre a stime tutt'altro che approssimative intorno all'ampiezza angolare del sole 5 . Ma anche la semplice procedura ideata per il calcolo dell'altezza delle piramidi poté sorprendere grandemente. A maggior ragione la previsione di una eclisse dovette strabiliare, specialmente se le cose fossero andate come lascia intendere Erodoto (in I 74), se cioè Talete poté asserire che l'eclisse si sarebbe verificata "in uno dei prossimi noviluni" avendo un motivo per asserirlo (e quindi per restringere il campo delle date possibili a uno o due giorni per ogni mese lunare). I punti che ho appena richiamato sono, in verità, congetturali da più di un punto di vista, tuttavia il loro insieme - ed è appena il caso di ricordare che, in realtà, l'insieme sarebbe molto più vasto - non manca di far pensare che la figura di Talete sia stata proprio eccezionale e il suo anomalo sapere tale da fare notizia.

Un altro ordine di considerazioni riguarda la qualifica di sophos. Conferire ufficialmente tale qualifica a uno, o eventualmente a sette personaggi in vista, poté equivalere a trasformare un epiteto già in uso in una vera e propria onorificenza. Il fatto che questo rito non si sia poi ripetuto e sia rimasto perciò un unicum contribuisce a rassicurarci sulla plausibilità della notizia. Osserviamo però che, una volta conclusa la fase delle onorificenze attribuite dagli ateniesi, non si affermarono particolari restrizioni nell'uso dell'epiteto. Eraclito ad es. parla, sia pure con una punta di sarcasmo, di

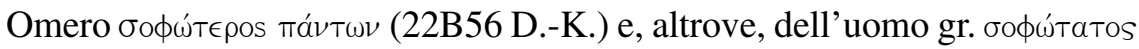
che, se paragonato alla divinità, si direbbe piuttosto una scimmia (22B83 D.-K.). Nemmeno il passo di Senofane su "noi sophoi" (elegia sullo sport =

5 Lo riferisce Apuleio in Florida 18.32-34 = 11A19 D.-K. = Th 178 Wöhrle. 
21B2 D.-K.) può dirsi particolarmente significativo ${ }^{6}$. Ciò significa che l'uso di tale epiteto rimase vincolato a un qualche atto pubblico unicamente nel caso dei "sette", e anche questa circostanza depone a favore dell'attendibilità della notizia. Invece l'ipotesi di un falso storiografico comporterebbe un accumulo eccessivo di invenzioni arbitrarie: il riferimento ad Atene, la scelta dell'anno, la natura del riconoscimento, il riferimento a Talete, la stessa costituzione di una sorta di 'collegio' dei sette sapienti, la stessa eventualità che Talete si sia recato ad Atene per l'occasione.

Congruente con quelli sopra esaminati è anche il dato relativo alla storia del tripode, che senza dubbio presenta elementi leggendari, ma sembra fondarsi anch'essa sull'avvenuto riconoscimento della superiore sophia di

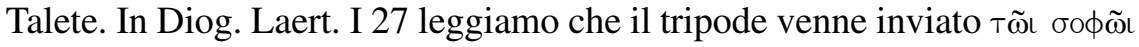
(ai sapienti) del popolo di Mileto. Ciò potrebbe significare che a Mileto c'era un intero gruppo di 'intellettuali' considerati sophoi dalla pubblica opinione. Si sarà trattato proprio di Talete e dei suoi allievi? Osserviamo inoltre che in questo testo Diogene Laerzio riferisce dapprima che il tripode venne offerto a Talete, poi ad altri e infine a Solone, il quale ultimo avrebbe rifiutato il dono, ritenendo che l'eccellenza nella sophia spettasse alla divinità. Subito dopo leggiamo che, secondo altre fonti, il tripode venne offerto a Talete, che lo rifiutò, poi ad altri, poi di nuovo a Talete, che infine lo inviò a Delfi, segnalando il privilegio di essere stato riconosciuto sophos 'dai Greci' (ossia non solo nella sua città) per ben due volte.

Pur avendo aspetti inequivocabilmente leggendari, la storia del tripode parte dal presupposto che Talete e i suoi allievi sarebbero stati dei buoni candidati alla qualifica di sophoi e che a Mileto i soli veri sophoi fossero proprio Talete e i giovani della sua cerchia. Vengono pertanto trasmesse due idee: (a) l'idea di una ricerca dei veri sophoi, (b) l'idea che a Mileto ci fossero, senza alcun dubbio, degli ottimi candidati (o almeno un ottimo candidato) a tale qualifica. Inoltre, se un buon candidato era disponibile

6 Ha senso addurre inoltre che, quando un intero gruppo di 'intellettuali' avvertì l'esigenza di presentarsi come sophistai, dietro la loro scelta ci poté essere l'aspirazione a caratterizzarsi meglio, a introdurre una certa enfasi, a sottolineare il carattere spiccato della loro sophia. Ora ciò implica che essi percepirono la qualifica di sophos come relativamente generica, relativamente povera di connotazioni, insomma come un epiteto un po' sbiadito, fors'anche perché la qualifica era stata nel frattempo estesa anche a figure che già in pieno V secolo poterono sembrare poco caratteristiche, come Cleobulo, Misone, Pittaco, Biante, personaggi ai quali non riusciamo (o almeno non riusciamo più) ad associare una precisa identità culturale. 
a Mileto e uno ad Atene, se il primo era Talete e il secondo Solone, ciò incoraggia a pensare che, all'epoca, una ricerca dei più veri sophoi venne effettivamente intrapresa. Si delinea insomma una realistica possibilità di pensare che la qualifica di sophos sia diventata, per qualche tempo, un epiteto prestigioso e che Talete sia stato il perno di questo tentativo di potenziare il significato di tale parola.

Abbiamo dunque buone ragioni per pensare che a Talete sia stata conferita la prima 'laurea honoris causa' e - dettaglio interessante - non una laurea in filosofia perché, come raramente viene ricordato, anche se la parola filosofia ha avuto una qualche circolazione nel V secolo, furono i Socratici per primi a pubblicare opere dichiaratamente filosofiche (i dialoghi) e, così facendo, a dare concretezza e contenuti a una parola che in precedenza (e se si eccettua il solo Socrate) aveva avuto usi poco più che occasionali. Talete e la generalità di quegli intellettuali che noi siamo soliti identificare come "presocratici" divennero filosofi (nel senso che cominciarono ad essere assimilati ai filosofi) solo nei decenni successivi ${ }^{7}$, mentre viventi ebbero, a quanto pare, soltanto la qualifica di sophoi (nonché, in maniera

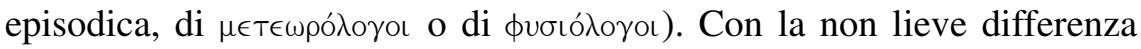
rappresentata dal fatto che, con ogni verosimiglianza, Talete, e solo Talete tra tutti i "presocratici", venne ufficialmente dichiarato sophos ad Atene.

Se, come pare, la notizia ha un fondamento storico, molte altre considerazioni sarebbero pertinenti, e si tratterebbe di considerazioni decisamente eterogenee. Si potrebbe prendere spunto da questa notizia per soffermarsi sulla passione greca per la competizione e la propensione ad apprezzare forme diverse di eccellenza, tendenza che sembra essersi manifestata in modo particolare proprio nel corso del VI secolo; ma attenzione meriterebbero inoltre gli indizi di sicura eccezionalità della figura di Talete anche in relazione agli altri sophoi di Mileto; sarebbe non meno pertinente interrogarsi sulle ragioni che possono aver determinato il processo di banalizzazione della figura di Talete, processo che ha caratterizzato gran parte della storiografia filosofica del Novecento. Su un altro piano si

7 In effetti l'uso di assimilare Talete e, più in generale, gli autori di scritti Peri physeos ai filosofi sembra essersi affermato intorno alla metà del IV secolo a.C. in ambiente platonico-aristotelico, dando luogo a un immediato consensus omnium che, come sappiamo, si è rivelato virtualmente irreversibile. In proposito v. Rossetti 2010 e la relazione che ho presentato al III Simpósio Internacional OUSIA de Estudos Clássicos sul tema: Physis. $O$ conceito de natureza na antiguidade, che ha avuto luogo a Rio de Janeiro per iniziativa della UFRJ nel giugno 2010. 
dovrebbe parzialmente ripensare il modo corrente di rappresentarci i Sette Sapienti. Rinuncio tuttavia a soffermarmi su questi aspetti ora, poiché queste pagine sono dedicate piuttosto all'accertamento di un fatto $^{8}$, dunque a un passaggio preliminare che non è il caso di confondere con questioni collaterali e derivate.

\section{Riferimenti bibliografici}

Moscarelli, E. , I quattro grandi Milesi, Napoli 2005.

O'Grady, P. , Thales of Miletus: The Beginnings of Western Science and Philosophy, Aldershot \& Burlington VT 2002.

Rossetti, L. , "I Socratici ' primi filosofi' e Socrate 'primo filosofo' “, in L. Rossetti \& A. Stavru (eds.), Socratica 2008, Bari 2010, 59-70.

SAssi, M. M., Gli inizi della filosofia: in Grecia, Torino 2009.

WöHrLe, G. (ed.), Die Milesier: Thales, Berlin 2009.

8 Ho effettuato una prima presentazione dei temi svolti in queste pagine nell'ambito del XVII Simposio Interdisciplinar de Estudos Grecoromanos di São Paulo sul tema Primeiros sábios, primeiros filósofos, promosso dalla PUC-SP nel maggio 2010. 
